\title{
Vision based Automatic line inspection Quadrotor Aircraft
}

\author{
Yang Yang ${ }^{1, a}$, Nannan Cheng ${ }^{2, b}$ \\ ${ }^{1}$ Department of Electrics Information Engineering, North China University of Technology, Beijing, \\ 100144, China \\ ${ }^{2}$ Department of Computer Science, North China University of Technology, Beijing, 100144, China \\ aemail: yangy_ncut@163.com, bemail:Cheng_nn@163.com
}

Keywords: quadrotor; Line inspection; vision; PIXHAWK; Raspberry Pi

\begin{abstract}
A vision based automatic line inspection quadrotor aircraft was designed. The aircraft was constructed and the algorithm was designed. PIXHAWK and Raspberry Pi were used as the dual control core of quadrotor, where PIXHAWK was used to complete the attitude control and Raspberry Pi captured the video to analyze the information of black guide line. And Raspberry Pi will send the control information to PIXHAWK and complete the aircraft control. Test Result shows that the aircraft has low cost, flexible application scene and strong adaptability to the field, which has great application value in some special environment.
\end{abstract}

\section{Introduction}

In recent years, with the continuous development of communication technology, sensor technology and control technology. Research on the quadrotor aircraft has become a hot spot in the field of Unmanned Aerial Vehicle (UAV). The quadrotor aircraft has the characteristics of small size, light weight, low cost, good safety and flexible. So it has been widely used in military and civilian areas.

Due to the constraint of natural conditions and environment, there are many problems in manual inspection field. The use of intelligent inspection devices has advantages of high efficiency, energy saving and time saving. So it is wildly used in some fields. Such as in power system, researchers conducted a wide range of exploration, designed some inspection device for substation, transmission lines [1, 2], which has developed a number of intelligent unmanned aerial vehicle intelligent inspection system [3, 4].

The key point of intelligent inspection is trajectory recognition and tracking. Currently, tracking methods including infrared method [5], passive RFID[6] and vision[7]. There are limits on the distance between the measured trajectory and sensor by using infrared method. And passive RFID need to add RFID tag while tracking, which will increase additional workload and cost. Vision based method need to add a CCD camera on the quadrotor, it has no other requirements for the trajectory and the environment. So, this paper selects a vision way to complete the automatic line inspection.

\section{Quadrotor aircraft design and control}

A. Hardware platform design

We need to build a quadrotor aircraft platform firstly, which includes three parts, quadrotor aircraft, communication module and ground station. Quadrotor aircraft can be guided by the intelligent algorithm to complete a full autonomous flight. The ground station can carry out real-time monitoring data of the aircraft, and the flight parameters are acquired through the communication module. Because of the small size and light weight of the aircraft, the calculation of the visual algorithm is relatively large, so the calculation ability, size, power consumption and quality of the processing unit are strictly required.

In order to meet the above requirement, this paper uses a dual processor mode to complete the flight control and image processing functions respectively. Quadrotor aircraft is shown in Figure 1, 
the main components including (1) a full carbon fiber body; (2) Emax 2213 935KV motor;(3) Hobbywind Skywalker 25A electrically adjustable; (4) PIXHAWK flight control; (5)8 inch blade; (6) Raspberry Pi. The overall weight of the aircraft is $1.38 \mathrm{~kg}$, effective load $0.5 \mathrm{~kg}$.

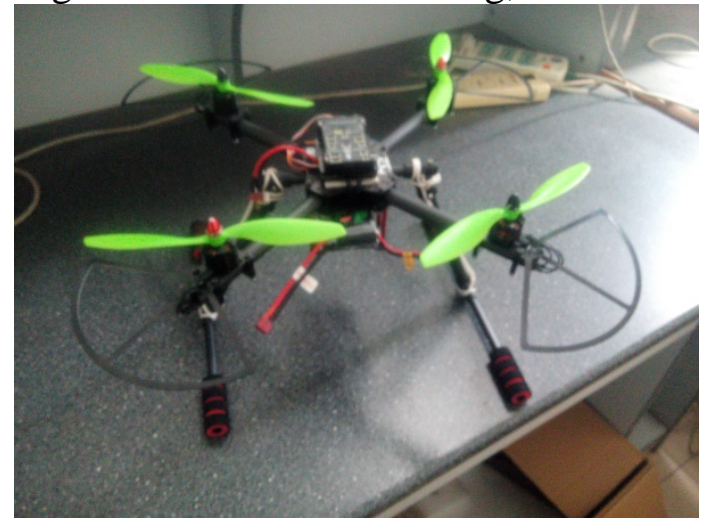

Fig.1. Quadrotor Aircraft

The attitude sensors are integrated in the PIXHAWK flight control. This module integrated three axis accelerometer, three axis gyroscope, three axis electronic compass and air pressure detection sensor, which meet the requirement of aircraft attitude solution. And the camera is installed on the raspberry to complete the image acquisition and analyzation.

B. Quadrotor aircraft control

Attitude solution and flight control is done by PIXHAWK, however, to complete the autonomous flight, image analysis is needed. And the Raspberry Pi will plan the path and adjust the attitude of the quadrotor to complete automatic tracking function. The key part is flight action setting and control signal transmission.

Raspberry Pi will send message to PIXHAWK through SBUS protocol, by controlling four values: throttle, steering, pitch and roll to control the attitude of the aircraft. Different values represent different degrees of output. From the beginning, the aircraft will complete different movement, and then through the addition delay to become a complete process, unlock, take off, flight, landing and locked.

The control signal of PIXHAWK is SBUS protocol. It is transmitted from raspberry Pi continuously. The protocol is 25 Byte long and is send every $7 \mathrm{~ms}$. Each Byte is organized as following: One Byte $=1$ startbit +8 databit +1 paritybit +2 stopbit. The baudrate is 100000 bit/s. And the highest bit is send first. The whole 25 bytes of the frame is [startbyte] [byte1] [byte2] .... [byte22] [flags][endbyte]

All the bytes include 16 channels, each channel will use 11 bits from the frame. For example, channel 1 uses 8 bits from data 1 and 3 bits from data2, channel 2 uses last 5 bits from data 2 and 6 bits from data3 etc.

\section{Line inspection algorithm}

Line inspection was implemented by visual method. Due to the limitation of size and power, the Mini USB in-line camera was connected to the raspberry Pi. The typical output format of camera is RGB, a maximum of 30 million pixels, 640x480 resolution.

A. Binarization

Because the color value distribution of RGB is much dispersed, and the correlation of three component is relatively high, which is not easy to identify the RGB threshold range, resulting in inaccurate trajectory identification. In this paper, HSI model is used as the main parameters, which can improve the accuracy of the identification. The Brightness determine the degree of saturation $S$, then according to the level of brightness, we adjust the value of saturation $S$ dynamically, so as to achieve the binarization of the image. As a result, the HSI model is used as the basis for the process of trajectory recognition, and select hue $H$ and saturation $S$ as the basis for the identification of the track. The pixels that satisfy the following constraints are the target trajectories: 
$H_{\min }<H<H_{\max }$

Where $H_{\min }$ is the lower threshold of the hue; $H_{\max }$ is the upper threshold.

$S_{\min }<S<S_{\max }$

Where $S_{\min }$ is the lower threshold of the saturation; $S_{\max }$ is the upper threshold.

B. Image denoising

Due to the effect of environment, the process of image capture will produce noise. Image noise will influent the detection and identification of trajectory, so image denoising is a prerequisite for the image processing. In this paper, median image filter is used for the image denoising. The idea is to sample the pixels in the range of image window $P(n-N) \ldots P(\mathrm{n}) \ldots P(\mathrm{n}+\mathrm{N})$, and take the median value, that is, the value of the $n$ pixel is:

$$
Y(n)=\operatorname{med}[\mathrm{p}(\mathrm{n}-\mathrm{N}) \ldots \mathrm{P}(\mathrm{n}) \ldots \mathrm{P}(\mathrm{n}+\mathrm{N})]
$$

Where med[] is the median value of all the value of pixels in selected image range in order of small to large. In practice, the image processing is already binarization images, so all the pixels have only two values 0 and 1 . The result will filter small noise and enlarge large noise. But the region of interest is basically unchanged and only need to remove the area of the largest area in the connected area, which is the desired trajectory.

$$
R O I=\max \left(\text { area }_{1}, \text { area }_{2} \ldots \text { area }_{n}\right)
$$

Where ROI is the area of interest, area $_{n}$ is the area of $\mathrm{n}$ connected pixels.

\section{Guide line processing}

Compared to car tracking, the quadrotor aircraft flight fast and has a certain height with the distance to the ground. And it has throttle, steering, pitch, and roll four values need to control. So the requirement for the speed and efficiency of image processing is relatively high. In order to simplify the control, we only controlled steering, pitch and roll three value. In the process of taking off, the throttle value is set constant, which will keep the aircraft fly at a constant height. The direction is controlled by steering, and the relative position is controlled by the value pitch and roll.

During flight, aircraft will encounter the following situations, go straight, turn left, turn right and cross (Figure 2). Algorithm is divided into position control and angle control, position control is to ensure the aircraft at the top of the guide line, and will not deviated from the guide line. The angle control the pitch angle of the aircraft, which makes the quadrotor track the trajectory. 


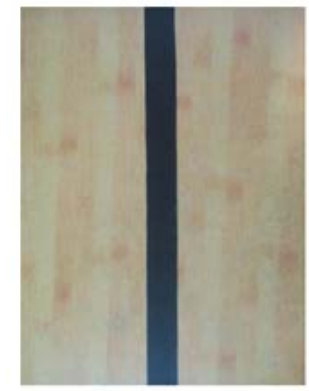

(a) straight line

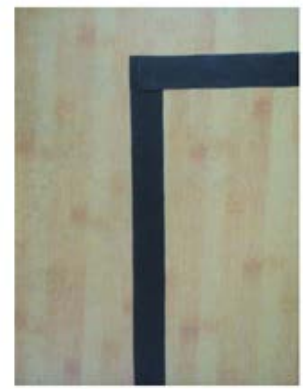

(c) turn right

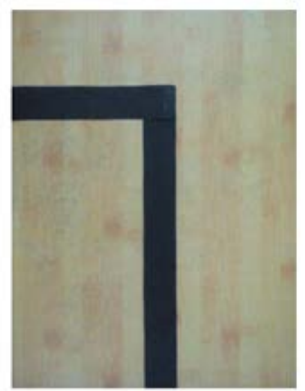

(b) turn left

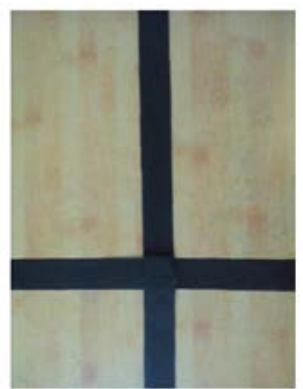

(d) cross

Fig.2. Guild Lines

The basic algorithm is as follows:

1) Find the region of interest, which is the trajectory of region, extract its center point $\left(\mathrm{x}_{\text {roi }}, \mathrm{y}_{\text {roi }}\right)$.

2) Using PID algorithm to calculate the error of the center point and the center of the image $\left(\mathrm{x}_{\text {image }}, \mathrm{y}_{\text {image }}\right)$. The PID algorithm is used to calculate the error, adjust the pitch and the roll to control the relative positon of the quadrotor and the trajectory.

3) Calculate the current deviation angle of quadrotor. Calculate the center coordinates in the image edge of the region of interest. Find the angle between the line connecting two center point and $y$ axis. Using this angle as patch angle, and using the PID algorithm to calculate the error and adjust the steering value to control the flight direction. The deviation angle is:

$$
\boldsymbol{\theta}=\arccos \left(\frac{a b s\left(\mathrm{y}_{1}-\mathrm{y}_{2}\right)}{\sqrt{\left(\mathrm{x}_{1}-\mathrm{x}_{2}\right)^{2}+\left(\mathrm{y}_{1}-\mathrm{y}_{2}\right)^{2}}}\right)
$$

Where $\theta$ is the deviation angle, $\left(\mathrm{x}_{1}, \mathrm{y}_{1}\right)$ and $\left(\mathrm{x}_{2}, \mathrm{y}_{2}\right)$ represent the center point coordinates of the image edge of region of interest, $a b s\left(\mathrm{y}_{1}-\mathrm{y}_{2}\right)$ is the width of region of interest. The calculation methods of straight line, turning left is shown in Figure 3. In the case of cross, the quadrotor is flying to a predetermined direction. 


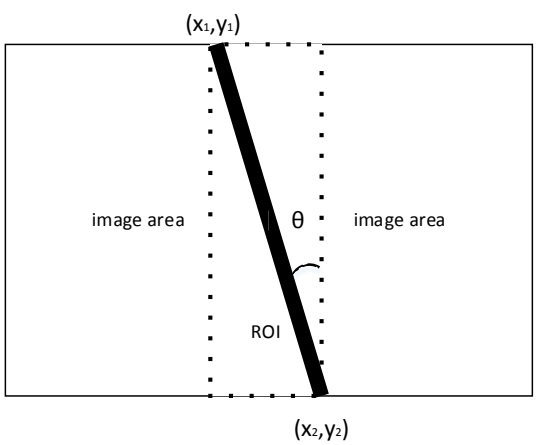

(a) Straight I ine

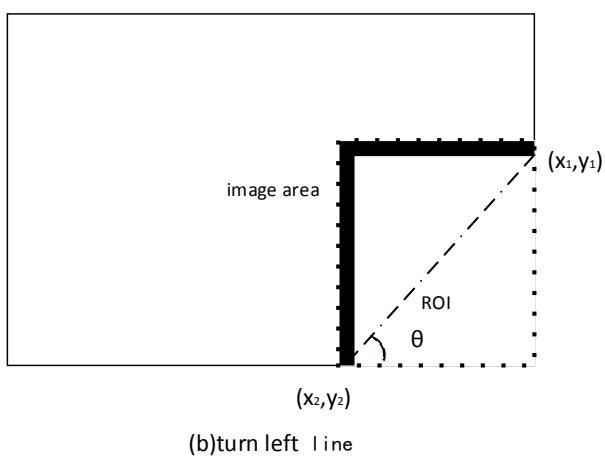

Fig.3. deviation angle

\section{Experimental Results}

In order to test the algorithm accuracy, we lay the black guide line in the laboratory, let the quadrotor fly under the same condition as a speed of $1 \mathrm{~m} / \mathrm{s}$. Tracking path including a $5 \mathrm{~m}$ long straight line, $45^{\circ}, 90^{\circ}, 135^{\circ}$ bend line and a cross. Test Results is shown in Table 1:

Table.3. Experiment result of Tracking

\begin{tabular}{llll}
\hline Path Type & Success time & Experiment time & Success rate \\
\hline Straight & 100 & 100 & $100 \%$ \\
$45^{\circ}$ & 92 & 100 & $92 \%$ \\
$90^{\circ}$ & 99 & 100 & $99 \%$ \\
$135^{\circ}$ & 98 & 100 & $98 \%$ \\
Cross & 98 & 100 & $98 \%$ \\
\hline
\end{tabular}

The results show that the algorithm has a high success rate at straight line, and at $90^{\circ}, 135^{\circ}$ and cross bend line, it doesn't make much of a difference, which can fulfill the function of tracking. While in $45^{\circ}$ bend situation, the track produced a turn back, which leads to a higher failure rate. But it also illustrate that the algorithm has a high success rate to the trajectory without a large turn back.

\section{Conclusion}

This paper designed an automatic line inspection quadrotor aircraft based on vision, which achieve accurate tracking of black guide line. Aircraft uses raspberry Pi and PIXHAWK as double core control unit. The flight control function was completed by PIXHAWK. Raspberry Pi connect camera and extract black guide line. The center coordinates and image center points were used to control the positon of the aircraft, and the center point coordinates of the region of interest at the image edge and its deviation of the ${ }^{y}$ axis were used to control the pitch. Test results show that the design of tracking aircraft has a high success rate, and has the advantages of low power consumption and low cost, which has important value and wide application prospect in some 
special environment.

\section{References}

[1] L. H. Zhou, Y. S. Zhang, Y. Sun. Development and application of intelligent substation inspection robot[J]. Automation of electric power system, 2011, 35(19):85-88.

[2] G. P. Wu, Y. Cao, Y. Pi. Self inspection robot and its application in high voltage and multi split transmission line[J]. Journal of Wuhan University, 2012,45(1);96-1-2.

[3] W. R. Cao, L. L. Zhu, J. D. Han. High Voltage Transmission Line Detection For UAV Based Routing Inspection[C]. International Conference on Advanced Intelligent Mechatronics, 2013.

[4] P. Tang, W. D. Jin, L. Chen. Visual Abnormality Detection Framework for Train-Mounted Pantograph Headline Surveillance[C]. 17th International conference on intelligent transportation systems(ITSC), 2014.

[5] Y. Wang, X. Jin, Q. A. Li. T-shaped infrared sensor design of the robot and complex trajectory tracking method[C]. IEEE Youth Conference on Information, Computing and Telecommunication, 2009.

[6] Yang, W. Y. Wu, M. Moniri. SLAM Algorithm for 2D Object Trajectory Tracking based on RFID Passive Tags[C]. IEEE International Conference on RFID,2008.

[7] S. Lee, J. H. Park, Virtual Trajectory in Tracking Control of Mobile Robots[C]. international conference on Advanced Intelligent Mechatronics (AIM 2003),2003. 